01

\title{
Расслаивающиеся полимерные растворы в микроразмерных порах: фазовые переходы, индуцированные деформацией пористого материала
}

\author{
(C) А.В. Шишулин, В.Б. Федосеев
}

Институт металлорганической химии им. Г.А. Разуваева РАН, 603137 Нижний Новгород, Россия

e-mail: Chichouline_Alex@live.ru

Поступило в Редакцию 31 июля 2019 г.

В окончательной редакции 31 июля 2019 г.

Принято к публикации 23 сентября 2019 г.

\begin{abstract}
В рамках термодинамического подхода рассмотрено влияние морфологии пор на фазовые равновесия в расслаивающихся жидких растворах в пористой матрице. На примере раствора олигомерных фракций полибутадиена и полистирола показано, что деформация матрицы, задающая геометрические характеристики пор, позволяет управлять взаимными растворимостями компонентов, объемами и термодинамической устойчивостью сосуществующих при равновесии фаз. Изменение геометрических характеристик пор в общем случае смоделировано введением параметра, соответствующего степени отклонения формы границ раздела от сферической. При этом характер зависимостей растворимостей компонентов от объема поры в порах различной формы отличаются, что объясняется реализацией различных механизмов понижения свободной энергии системы. При значительных деформациях матрицы расслаивание в микроразмерных порах подавляется, и устойчивыми становятся любые составы растворов вплоть до эквимолярного.
\end{abstract}

Ключевые слова: пористые материалы, фазовые превращения, core-shell, деформация, расслаивание.

DOI: 10.21883/JTF.2020.03.48917.292-19

\section{Введение}

Микро- и наноструктурные композиционные материалы на основе пористых матриц с включениями жидких [1-3] или твердых [4,5] растворов имеют широкий спектр технологических приложений $[3,4,6-10]$ (от нанокомпозиционных бронепакетов с повышенной энергопоглощающей способностью [3] и сред с аномальным характером распространения фононов [6] до материаловмоделей биологических тканей [9] и материалов со специфическими свойствами, обусловленными квантоворазмерными эффектами [4]) и являются актуальным предметом исследования на стыке физики конденсированного состояния, физической химии и технологии материалов. Ряд специфических свойств таких композитов в значительной степени обусловлены отклонением свойств материалов в микро- и наноструктурах (микро- и наноразмерных порах [1,2,7,11], тонких пленках и слоях $[11,12]$, каплях малого объема $[13,14])$, наночастицах [15-22] и зернах поликристаллического материала [23]) от свойств макроскопических систем. В частности, термодинамические параметры (температуры, давления и т.д.) фазовых переходов [2,10,13-20,22], равновесный фазовый состав в структурах малого объема $[1,10,14,17,20-23]$, а также их термодинамическая стабильность могут существенно отличаться от аналогичных характеристик для макросистем. Кроме того, особенностью систем малого объема является возможность образования нехарактерных для материалов в макроско- пическом состоянии метастабильных фаз [2,14,20,21], а также сложная (в том числе и колебательная) динамика фазовых переходов [24].

В жидких расслаивающихся растворах в капиллярах или порах некоторые специфические особенности могут быть связаны, например, с капиллярным расслаиванием [25] (изменением равновесного состава раствора в результате преимущественной адсорбции одного из компонентов на стенке поры), а в нанокристаллах например, с изменением концентрации и подвижности дефектов кристаллической структуры [26], энергии их образования и энергии активации диффузии [27]. Существенная же часть эффектов $[1,2,10,12,14-23]$ (в том числе наблюдавшихся экспериментально $[13,14,23,24])$ связана с возрастанием роли границ раздела при уменьшении характерного размера системы и может быть, наряду с некоторыми иными особенностями [25-27], описана и интерпретирована в рамках термодинамического подхода. Увеличение относительного энергетического вклада границ раздела приводит к смещению положений термодинамического равновесия, что на фазовых диаграммах проявляется в сдвигах и деформациях характеристических линий, отображающих изменения взаимных растворимостей, объемных долей сосуществующих фаз и температур фазовых переходов, причем величины изменений зависят от размера системы $[1,2,10,13-23,28]$, ее формы $[1,2,10,18-22]$ и термодинамических характеристик ее межфазных границ [28]. При описании процессов и свойств систем малого объема справочные 
данные о диаграммах состояния требуют существенного уточнения с учетом подобных эффектов.

В системах, представленных каплями жидких расслаивающихся растворов (например, смесями олигомерных фракций полибутадиена и полистирола, находящихся в жидком состоянии при комнатной температуре $[29,30])$ в микро- и наноразмерных порах, подобных описанным в $[1-3,7,9]$, форма поры, оказывающая влияние на равновесный фазовый состав, может гибко задаваться плавными деформациями матрицы при различных вариантах приложения нагрузки. Расслаивание жидких растворов в порах зачастую приводит к образованию структуры „ядро-оболочка“ (core-shell) [1,2,31,32]. Форма поры, определяющаяся степенью деформации матрицы, может быть охарактеризована параметрически с использованием различных подходов [1,2,10,20-22,33-35], включая методы фрактальной геометрии [1,10,21,22,33].

\section{Термодинамическая модель фазовых равновесий в бинарных расслаивающихся жидких растворах в порах деформируемой матрицы}

Объектами моделирования являются капли эквимолярного состава жидкого расслаивающегося раствора олигомеров полибутадиена (PBD) и полистирола (PS) в порах деформируемой матрицы при температуре $T=300$ К. Согласно $[29,36]$, в макроскопической системе компоненты данной смеси не образуют непрерывного ряда жидких растворов, расслаиваясь с верхней критической температурой растворения (ВКТР) $\sim 420 \mathrm{~K}$. Предполагается, что при расслаивании в поре образуется единичное сферическое включение жидкого раствора, окруженное слоем жидкого раствора другого состава (core-shell-структура). Поры в недеформированной матрице имеют сферическую форму; и без потери общности дальнейших выводов положим, что при деформации они приобретают форму эллипсоидов (рис. 1), при этом

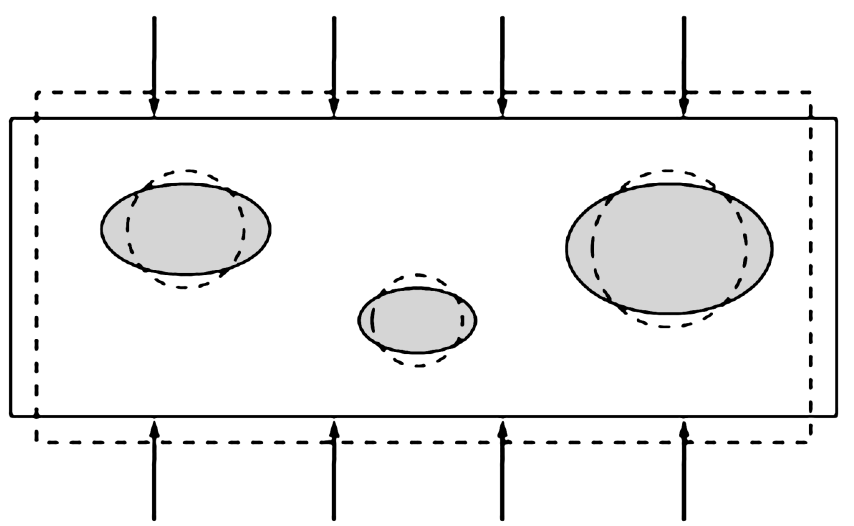

Рис. 1. К постановке задачи: раствор полимеров в порах деформируемой матрицы; штрихом показана недеформированная матрица со сферическими порами, сплошной линией продеформированная матрица с порами в форме эллипсоидов. сжимаемость жидкости, согласно [3], не учитывается. Для описания формы поры подобно [1,2,10,20-22] используем коэффициент формы поры $k$, численно равный отношению площади поверхности эллиптической поры в продеформированной матрице $A$ к площади поверхности поры до деформации $A_{0}, k=A / A_{0}$. Если смесь находится в двухфазном состоянии с конфигурацией core-shell, то геометрические характеристики системы описываются выражениями

$$
V_{j}=\sum n_{i j} V_{i}, \quad A_{c}=4 \pi\left(\frac{3}{4 \pi} V_{c}\right)^{2 / 3}, \quad A_{s}=k \pi d^{2},
$$

где $d$ - диаметр поры до деформации матрицы (эффективный диаметр поры); $V_{j}$ - объем фазы $j ; j=c, s$; индексам $c$ и $s$ соответствуют core- и shell-фазы; $n_{i j}$ и $V_{i}$ - число молей компонента $i$ в фазе $j$ и мольный объем компонента $i, i=1,2$; индексам 1 и 2 отвечают полибутадиен и полистирол соответственно; $A_{c}$ и $A_{s}$ - площади поверхностей core- и shell-фаз. Мольные объемы компонентов оценивались аналогично [29,37], согласно аддитивной схеме А. Бонди [38], путем суммирования вкладов в объем молекулы от каждого структурного элемента [29] и равны $V_{1}=1910 \mathrm{~cm}^{3} / \mathrm{mol}$, $V_{2}=1450 \mathrm{~cm}^{3} / \mathrm{mol}$. Молекулярные массы рассматриваемых олигомеров PBD и PS составляют $M_{1}=2655$ и $M_{2}=2400$ u.a.m.u. соответственно [29].

Условия сохранения вещества при фазовом превращении в закрытой двухкомпонентной системе связывают объем частицы, суммарное количество вещества в системе $n$, число молей каждого из компонентов $n_{i}$ и концентрации $x_{i j}$ компонентов $i$ в фазах $j$ :

$$
\begin{gathered}
\frac{1}{6} \pi d^{3}=n_{1} V_{1}+n_{2} V_{2}, \quad n_{1}=n_{2}=0.5 n, \quad n_{i}=n_{i c}+n_{i s} \\
x_{i j}=\frac{n_{i j}}{n_{1 j}+n_{2 j}} .
\end{gathered}
$$

Критерием равновесного состояния системы является минимум функции Гиббса с учетом энергетического вклада всех границ раздела

$$
\begin{aligned}
g=\left(n_{1 c}+n_{2 c}\right) G\left(x_{1 c}, T\right) & +\left(n_{1 s}+n_{2 s}\right) G\left(a_{1 s}, T\right) \\
& +\sigma_{s} A_{s}+\sigma_{c s} A_{c},
\end{aligned}
$$

где $\sigma_{s}, \sigma_{c s}$ - поверхностные натяжения на внешней $(-s h e l l)$ и на внутренней (core-shell) границах раздела.

Функция Гиббса рассматриваемой системы в области жидких растворов получена в рамках модели ФлориХаггинса [29,37], традиционно записываемой через объемные доли компонентов [39]:

$$
\begin{gathered}
G_{j}\left(\varphi_{1 f}, T\right)=R T\left(\frac{\rho_{1}}{M_{1}} \varphi_{1 f} \ln \varphi_{2 f}+\chi_{12}^{f} \varphi_{2 f}\right), \\
\varphi_{1 f}=\frac{n_{1 f} V_{1}}{V_{f}}, \quad \varphi_{2 f}=1-\varphi_{1 f},
\end{gathered}
$$

где $\varphi_{i f}$ - объемная доля компонента $i$ в фазе $f$; $\rho_{i}$ — „плотность“ мономерного сегмента каждого из 
компонентов, оцениваемая как отношение молекулярной массы мономера к объему его молекулы [29] (последний рассчитывался также в соответствии со схемой А. Бонди [38]); $\chi_{12}^{f}$ - коэффициент Флори-Хаггинса для фазы $f$, вычисляемый согласно [29]:

$$
\begin{aligned}
& \chi_{12}^{f}=0.0043-7.1 \cdot 10^{-6} T+\left(0.0003-6.9 \cdot 10^{-7} T\right) \\
& \times\left(\varphi_{1 f}-\varphi_{2 f}\right)+\left(0.000324-6.4 \cdot 10^{-7} T\right)\left(\varphi_{1 f}-\varphi_{2 f}\right)^{2} .
\end{aligned}
$$

Температурные зависимости поверхностных натяжений чистых полибутадиена и полистирола получены в [40]; при $T=300 \mathrm{~K}$ они составляют $\sigma_{1} \approx 45.63 \mathrm{~mJ} / \mathrm{m}^{2}, \quad \sigma_{2} \approx 41.86 \mathrm{~mJ} / \mathrm{m}^{2}$. В соответствии c $[1,2]$ (и ссылки в [1]), поверхностные натяжения жидких растворов органических компонентов на внешней (-shell) границе описываются в линейном приближении: $\sigma_{s}(x)=\sigma_{2}\left(1-x_{2}\right)+\sigma_{1} x_{1 s}$. Для оценки поверхностного натяжения внутренней (core-shell) границы $\sigma_{c s}$ используется разложение поверхностного натяжения каждого из компонентов на несколько составляющих [40], и, как показано в [41], для обоих компонентов системы доля составляющей, связанной с дисперсионными взаимодействиями, превышает $80 \%$; расчет величины $\sigma_{c s}$ осуществляется в рамках модели Ф. Фоукса [42]:

$$
\begin{gathered}
\sigma_{c s}=\sigma_{c}+\sigma_{s}-\sqrt{\sigma_{c}^{d} \sigma_{s}^{d}}, \sigma_{c}(x)=\sigma_{2}\left(1-x_{2 c}\right)+\sigma_{1} x_{1 c} \\
\sigma_{f}^{d}(x)=\sigma_{2}^{d}\left(1-x_{2 f}\right)+\sigma_{1}^{d} x_{1 f}
\end{gathered}
$$

Оценки величин дисперсионных составляющих $\sigma_{i}^{d}$ поверхностных натяжений чистых компонентов получены в [41] и при рассматриваемой температуре составляют $\sigma_{1}^{d} \approx 38.08 \mathrm{~mJ} / \mathrm{m}^{2}, \sigma_{2}^{d} \approx 36.88 \mathrm{~mJ} / \mathrm{m}^{2}$.

\section{Результаты моделирования и обсуждение}

Система с core-shell-структурой имеет два термодинамически равновесных гетерогенных состояния (минимума функции Гиббса (3)), различающихся взаимным расположением сосуществующих жидких растворов. В макроскопической системе, где энергетический вклад границ раздела мал, свободная энергия обоих состояний одинакова, а составы сосуществующих фаз соответствуют справочным данным [29,36]. С уменьшением размера системы рост вклада энергии межфазных границ приводит к смещению минимумов функции Гиббса, в результате чего равновесные составы и объемы сосуществующих фаз в обоих core-shell-cocтояниях отличаются от справочных значений для макросистем, различаются между собой и зависят от объема и площади внутренней поверхности поры (степени деформации матрицы). При этом состояние, где shell-фаза образована раствором на основе PBD, имеет при $T=300 \mathrm{~K}$ бо́льшую энергию межфазных границ и является метастабильным (соответствует локальному минимуму функции Гиббса (3)).

Как показано на рис. 2, $a$, в состоянии с раствором PS в PBD в положении shell-фазы уменьшение объема поры и деформация матрицы (увеличение коэффициента формы поры $k$ ) сопровождаются заметным повышением растворимости полистирола в полибутадиене. Например, в порах сферической формы (в недеформированной матрице) растворимость PS составляет: в макросистеме $\sim 4.18$ mol.\%; при диаметрах пор 350 и $250 \mu \mathrm{m}-$ $\sim 8.70$ и $\sim 12.95 \mathrm{~mol} . \%$ соответственно (растворимость полибутадиена в полистироле также изменяется, снижаясь в пределах на $\sim 1 \mathrm{~mol} \%$ ). При этом деформация поры диаметром $250 \mu \mathrm{m}$, сопровождающаяся ростом до $k=1.5$, вызывает увеличение растворимости полистирола в полибутадиене до $\sim 20.03 \mathrm{~mol} \%$. Доля объема, занимаемого core-фазой в core-shell-структуре, в свою очередь, падает при уменьшении диаметра поры и росте $k$ (рис. $2, b$ ). Например, в недеформированной матрице объемная доля core-фазы, составляя $\sim 42.8$ vol.\% в макросистеме, уменьшается до $\sim 39.80$ vol.\% в поре диаметром $350 \mu \mathrm{m}$ и до $37.4 \mathrm{vol} . \%$ - в поре диаметром $250 \mu \mathrm{m}$. В последнем случае рост $k$ от 1.00 до 1.50 приводит к дополнительному падению доли core-фазы до $\sim 33$ vol. $\%$.

В состоянии, где shell-фаза образована раствором PBD в PS, закономерности отличаются большей сложностью. Растворимость полибутадиена в полистироле в порах любого объема монотонно уменьшается при деформации матрицы (рис. 3,a); например, в сферической поре диаметром $40 \mu \mathrm{m}$ растворимость PBD составляет $\sim 2.38 \mathrm{~mol} \%$, а в поре того же объема с $k=1.50-\sim 1.12 \mathrm{~mol} . \%$. При этом реакция растворимости полибутадиена в полистироле на изменение объема поры различна для пор разной формы: для сферических и близких к сферическим пор растворимость при уменьшении объема повышается (составляя, например, $\sim 2.42$ и $\sim 2.67 \mathrm{~mol} \%$ в порах диаметром 50 и $30 \mu \mathrm{m}$ соответственно при $k=1.00)$. Однако при значительных деформациях матрицы порам меньшего объема соответствует меньшее значение растворимости (например, при $k=1.5$ растворимость $\mathrm{PBD}$ составляет $\sim 1.20$ и $\sim 0.94 \mathrm{~mol} \%$ для $d=50$ и $30 \mu \mathrm{m}$ соответственно). Более детально это показано на рис. $3, b$, где приведены зависимости растворимости от эффективного диаметра поры для различных степеней деформации матрицы от $k=1.00$ до $k=1.50$. В интервале $1.00<k<1.15$ с уменьшением объема поры растворимость растет, в области $k>1.15$ - уменьшается. Линия, соответствующая $k=1.15$, служит своего рода „сепаратрисой“ между этими областями - при данном значении $k$ растворимость практически не зависит от размера поры и близка к макроскопическому значению (штрих на рис. $3, b$ ).

Еще одним эффектом является вызываемое деформацией матрицы подавление расслаивания в поре. В состоянии, где shell-фаза представляет собой раствор PS в PBD (метастабильном), при $d=250 \mu \mathrm{m}$ деформация 

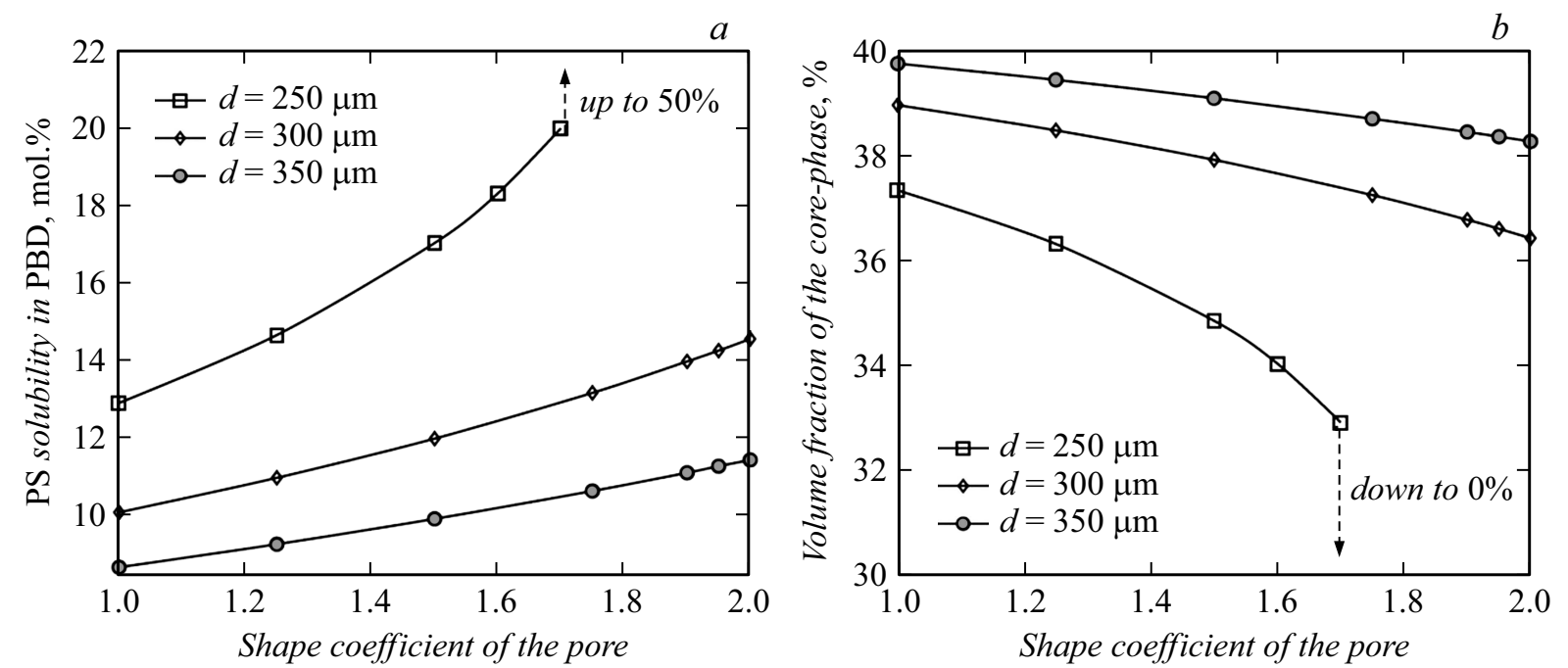

Рис. 2. Зависимость растворимости $\mathrm{PS}$ в $\mathrm{PBD}(a)$ и объемной доли соге-фазы $(b)$ от размера и коэффициента формы поры $k$ для состояния, где shell-фаза образована раствором на основе PBD. Значения растворимости PS в PBD и объемной доли core-фазы для макросистем составляют $\sim 4.18$ и $\sim 42.8$ vol.\% соответственно.
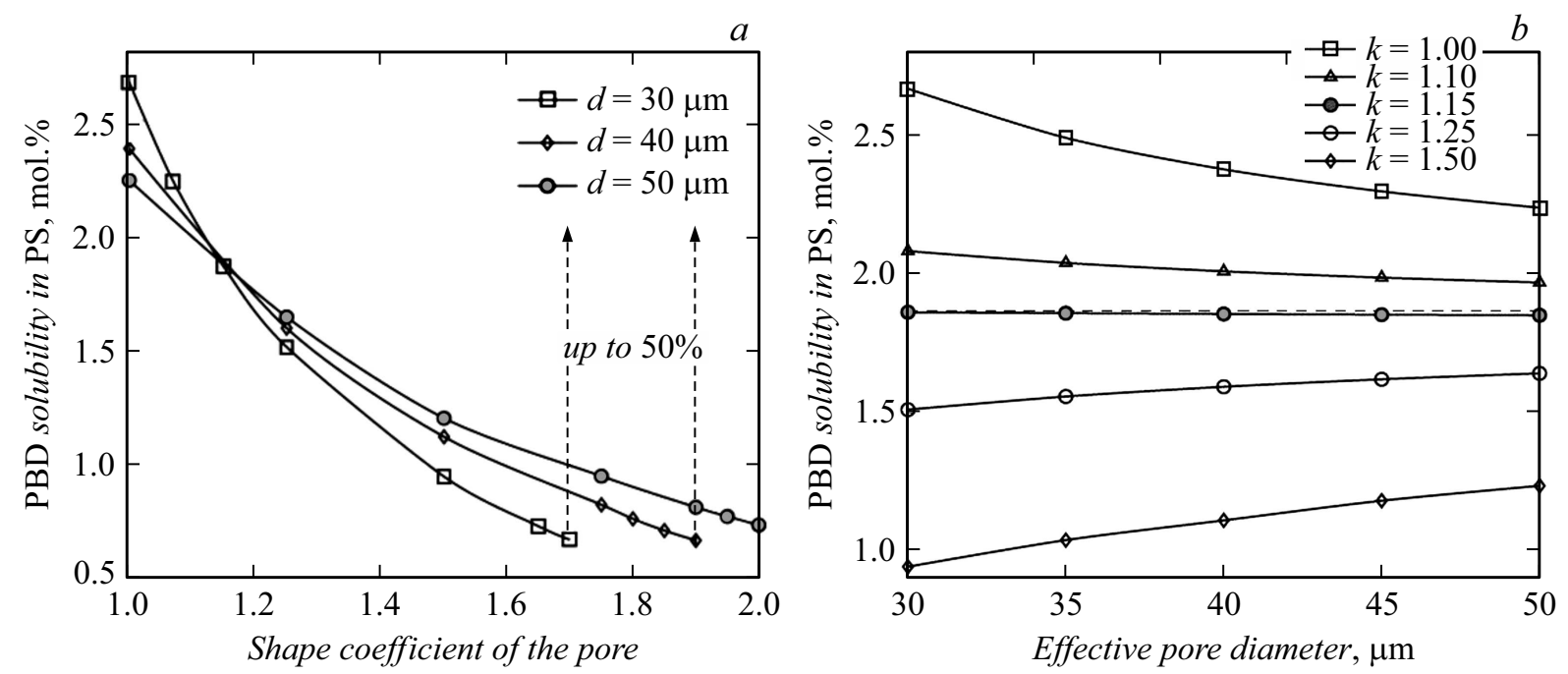

Рис. 3. Зависимости растворимости полибутадиена в полистироле от коэффициента формы $(a)$ и ее размера $(b)$ для состояния, где shell-фаза образована раствором на основе PS. Горизонтальным штрихом $(b)$ показана растворимость в системе макроскопического размера.

матрицы с $k>1.5$ делает расслаивание раствора в поре термодинамически невыгодным. В этом случае исчезает локальный минимум функции Гиббса (3), отвечающий метастабильному гетерогенному состоянию, и метастабильным состоянием становится гомогенное без образования внутренней межфазной границы (т.е. локальный минимум функции Гиббса в этом случае соответствует точке $x_{1 c}=x_{2 c}=0$; это показано штриховыми линиями со стрелками на рис. 2). При уменьшении объема поры гомогенизация в метастабильном состоянии происходит при все меньших деформациях матрицы, и при $d<100 \mu \mathrm{m}$ метастабильное гомогенное состояние заменяет гетерогенное метастабильное уже в сферических порах (и оно существует наряду с гетеро- генным состоянием, соответствующим глобальному минимуму свободной энергии). В состоянии, где shell-фаза образована раствором PBD в PS, в порах с $d=40 \mu \mathrm{m}$ расслаивание подавляется при $k>1.90$, с $d=30 \mu \mathrm{m}-$ при $k>1.70$; а с $d<15 \mu \mathrm{m}$ гомогенное состояние соответствует глобальному минимуму функции Гиббса и в сферических порах. В этих случаях глобальный и локальный минимумы функции Гиббса „сливаются“, и гомогенное состояние становится единственным возможным в системе.

Отклонения равновесного фазового состава системы от макроскопических значений и подавление расслаивания являются следствием понижения системой свободной энергии, которое реализуется посредством трех 
различных механизмов (при этом энергетический выигрыш, связанный с уменьшением энергии границ раздела, превышает „проигрыш“, связанный с формированием пересыщенных по сравнению с макроскопическими значениями $[29,36]$ растворов):

а) путем переноса вещества в shell-фазу (обоих компонентов или преимущественно компонента с бо́льшим мольным объемом (PBD)), приводящего к уменьшению площади внутренней межфазной границы;

б) путем преимущественного обогащения shell-фазы компонентом с меньшим поверхностным натяжением (PS), приводящего к уменьшению энергетического вклада внешней границы раздела (энергетический вклад внутренней core-shell-границы (7) существенно меньше по величине и менее чувствителен к составу сосуществующих фаз);

в) путем сохранения гомогенного состояния без образования внутренней межфазной границы.

При малых $k(1.00<k<1.15)$, когда форма поры близка к сферической, в состоянии с core-фазой, образованной раствором на основе полистирола, реализуется механизм (а). В этом случае при уменьшении объема частицы растет доля полибутадиена в shell-фазе, а объемная доля core-фазы снижается. В несферических порах при $k>1.15$, когда площадь внешней межфазной границы велика, происходит смена механизма, и реализуется механизм (б): уменьшение объема поры приводит к росту доли полистирола в shell-фазе. При постоянном объеме поры деформация матрицы, приводящая к увеличению площади поверхности поры, также вызывает реализацию механизма (б) (рис. 3,a). При $k=1.15$ два конкурирующих механизма (а) и (б) „компенсируют“ друг друга, и поэтому растворимость полибутадиена слабо чувствительна к изменению объема поры и практически не отличается от макроскопического значения. В состоянии с core-фазой, образованной раствором на основе полибутадиена, механизм (а) реализуется при любых объемах поры и степенях деформации матрицы. В порах малых размеров с высокими $k$ реализуется механизм (в), расслаивание „подавляется“ (это соответствует падению ВКТР раствора в поре ниже температуры $T=300 \mathrm{~K}[2,10,14,17,20,22])$.

Следует отметить, что полученные ранее оценки $[1,10,17,20-22,28,33]$ для ряда простых неорганических систем и водных растворов с органическим компонентом позволяют ожидать заметных изменений температур фазовых переходов и фазовых составов при модификации геометрических параметров системы, как правило, лишь в случаях, когда характерный размер системы не превышает сотен нанометров. Как показано выше (рис. 2,3), в случае полимерных растворов такие эффекты реализуются в области размеров, больших на 3-4 порядка, и изменения растворимостей могут иметь немонотонный характер $[1,2]$. Также при расслаивании растворов в поре следует ожидать проявления характерных для систем ограниченного объема закономерностей, связанных с зависимостью взаимных растворимостей компонентов от исходного состава системы (данные закономерности рассмотрены в [43]).

Выполненные термодинамические оценки демонстрируют возможность создания систем, где число, составы и термодинамическая устойчивость фаз (а также весь комплекс свойств, связанных с равновесным фазовым составом) задаваемы и управляемы путем простых внешних воздействий (например, деформацией матрицы). Многовариантность устойчивых состояний системы $[2,10,17,20-22]$ и возможность управляемого перехода между этими состояниями [2,24,44] делают полученные закономерности актуальными при создании и моделировании адаптивных мультистабильных систем, например, мемристивных устройств [45], а также соответствующих элементов нейронных сетей [46].

\section{Заключение и выводы}

Проведенный термодинамический анализ влияния геометрических характеристик пор (задаваемых их объемом и деформацией пористой матрицы) на фазовые равновесия в расслаивающихся внутрипоровых растворах на примере системы полибутадиен-полистирол приводит к следующим выводам:

1) в микроскопических порах равновесный состав и объем сосуществующих при расслаивании растворов фаз являются функцией геометрических характеристик поры. В образующейся при расслаивании core-shellструктуре возможно два устойчивых гетерогенных состояния, одно из которых метастабильно, при этом для различных состояний core-shell-структуры реакции взаимных растворимостей компонентов и объемов фаз на изменение объема и формы поры могут отличаться, что объясняется существованием нескольких различных механизмов понижения свободной энергии системы путем межфазного перераспределения компонентов;

2) в порах малого объема или при сильных деформациях матрицы распад раствора становится термодинамически невыгодным, и устойчивым становится гомогенное состояние раствора любого состава вплоть до эквимолярного. При этом подобные эффекты (индуцированные деформацией изменения взаимных растворимостей и гомогенизация) становятся заметными при эффективных диаметрах пор порядка сотен нанометров и менее.

\section{Финансирование работы}

Работа выполнена в соответствии с государственным заданием ИМХ РАН, а также при поддержке Российского фонда фундаментальных исследований (проект 18-08-01356-a).

\section{Конфликт интересов}

Авторы заявляют, что у них нет конфликта интересов. 


\section{Список литературы}

[1] Shishulin A.V., Fedoseev V.B. // J. Mol. Liq. 2019. Vol. 278. P. 363. DOI: 10.1016/j.molliq.2019.01.050

[2] Шишулин А.В., Федосеев В.Б. // Письма в ЖТФ. 2019. T. 45. Вып. 14. C. 10-12. [Shishulin A.V., Fedoseev V.B. // Tech. Phys. Lett. 2019. Vol. 45. N 7. P. 697-699.] DOI: $10.1134 / \mathrm{S} 1063785019070289$

[3] Брагов А.М., Игумнов Л.А., Константинов А.Ю., Ломунов А.К., Антонов Ф.К., Моссаковский П.А. // Письма в ЖТФ. 2014. Т. 40. Вып. 20. С. 8287. [Bragov A.M., Igumnov L.A., Konstantinov A.Yu., Lomunov A.K., Antonov F.K., Mossakovskii P.A. // Tech. Phys. Lett. 2014. Vol. 40. N 10. P. 923-925.]

DOI: $10.1134 / \mathrm{S} 1063785014100186$

[4] Ивичева С.Н., Каргин Ю.Ф., Шворнева Л.И., Куцев С.В., Юрков Г.Ю. // Неорган. матер. 2012. Т. 48. № 3. С. $346-$ 354. [Ivicheva S.N., Kargin Yu.F., Shvorneva L.I., Kutsev S.V., Yurkov G.Yu. // Inorg. Mater. 2012. Vol. 48. N 3. P. 289-297.] DOI: $10.1134 / \mathrm{S} 0020168512030065$

[5] Львов П.Е., Светухин В.В. // ФТТ. 2013. Т. 55. Вып. 11. С. 2256-2261. [L'vov P.E., Svetukhin V.V. // Phys. Solid State. 2013. Vol. 55. N 11. P. 2374-2380.] DOI: $10.1134 / \mathrm{S} 1063783413110140$

[6] Sato A., Knoll W., Pennec Y., Djafari-Rouhani B., Fytas G., Steinhart M. // J. Chem. Phys. 2009. Vol. 130. P. 11102. DOI: $10.1063 / 1.3096972$

[7] Acosta C., Pérez-Esteve E., Fuenmayor C.A., Benedetti S., Cosio M.S., Soto J., Sancenón F., Mannino S., Barat J., Marcos M.D., Martínez-Máñez R. // ACS Appl. Mater. Interfaces. 2014. Vol. 6. N 9. P. 6453-6460. DOI: $10.1021 / \mathrm{am} 405939 \mathrm{y}$

[8] Li Y., Zhang X., Manyalo J.M., Tian Z., Ji J. // J. Mol. Liq. 2019. Vol. 277. P. 577-583.

DOI: $10.1016 /$ j.molliq.2018.12.111

[9] Federico S., Herzog W. // Intern. J. Solid. Structur. 2008. Vol. 45. P. 2160-2172. DOI: 10.1016/j.ijsolstr

[10] Шишулин А.В., Федосеев В.Б., Шишулина А.В. // ЖТФ. 2019. Т. 89. Вып. 4. С. 556-561. [Shishulin A.V., Fedoseev V.B., Shishulina A.V. // Tech. Phys. 2019. Vol. 64. N 4. P. $512-517$.]

DOI: $10.1134 / \mathrm{S} 1063784219040200.2007 .11 .014$

[11] Бараш Ю.С., Нотыч О.И. // ЖЭТФ. 1990. Т. 98. № 2. C. 542. [Barash Yu.S., Notych O.I. // JETP. 1990. Vol. 71. N 2. P. 301-307.]

[12] Самсонов В.М., Сдобняков Н.Ю., Самсонов М.В., Соколов Д.Н., Новожилов Н.В. // Поверхность. Рентген., синхротр. и нейтрон. исслед. 2015. № 8. С. 7680. [Samsonov V.M., Sdobnyakov N.Yu., Samsonov M.V., Sokolov D.N., Novozhilov N.V. // J. Surf. Invest.: X-ray, Synchrotron Neutron Tech. 2015. Vol. 9. N 4. P. 831-835.] DOI: $10.1134 / \mathrm{S} 1027451015040333$

[13] Титаева Е.К., Федосеев В.Б. // Кристаллография. 2014. T. 59. № 3. C. 484-488. [Titaeva E.K., Fedoseev V.B. // Cryst. Rep. 2014. Vol. 59. N 3. P. 437-441.] DOI: $10.1134 / \mathrm{S} 1063774514030195$

[14] Федосеев В.Б., Шишулин А.В., Титаева Е.К., Федосеева Е.Н. // ФТТ. 2016. Т. 58. Вып. 10. С. 2020-2025. [Fedoseev V.B., Shishulin A.V., Titaeva E.K., Fedoseeva E.N. // Phys. Solid State. 2016. Vol. 58. N 10. P. 2095-2100.] DOI: $10.1134 / \mathrm{S} 1063783416100152$
[15] Sim K., Lee J. // J. Alloys Compd. 2014. Vol. 590. P. 140-146. DOI: $10.1016 /$ j.jallcom.2013.12.101

[16] Monji F., Jabbareh M.A. // CALPHAD: Computer Coupling Phase Diagrams Thermochem. 2017. Vol. 58. P. 1-5. DOI: $10.1016 /$ j.calphad.2017.04.003

[17] Шишулин А.В., Федосеев В.Б. // Неорган. материалы. 2018. Т. 54. № 6. С. 574-578. [Shishulin A.V., Fedoseev V.B. // Inorg. Mater. 2018. Vol. 54. N 6. P. 546549.] DOI: $10.1134 / \mathrm{S} 0020168518050114$

[18] Guisbiers G., Mendoza-Pérez R., Bazán-Díaz L., MendozaCruz R., Velázquez-Salazar J.J., Yakamán M.J. // J. Phys. Chem. 2017. Vol. 121. N 12. P. 6930-6939. DOI: $10.1021 /$ acs.jpcc.6b09115

[19] Cui M., Lu H., Jiang H., Cao Z., Meng X. // Sci. Rep. 2017. N 7. P. $1-10$. DOI: $10.1038 /$ srep41990

[20] Федосеев В.Б., Шишулин А.В. // ФТТ. 2018. Т. 60. Вып. 7. С. 1382-1388. [Fedoseev V.B., Shishulin A.V. // Phys. Solid State. 2018. Vol. 60. N 7. P. 1398-1404.] DOI: $10.1134 / \mathrm{S} 1063783418070120$

[21] Шишулин А.В., Федосеев В.Б. // Кинет. катал. 2019. T. 60. № 3. C. 334-338. [Shishulin A.V., Fedoseev V.B. // Kinet. Catal. 2019. Vol. 60. N 3. P. 315-319.] DOI: $10.1134 / \mathrm{S} 0023158419030121$

[22] Shishulin A.V., Potapov A.A., Fedoseev V.B. Advances in Artificial Systems for Medicine and Education II / Ed. by Z. Hu, S. Petoukhov, M. He. Springer, 2020. P. 405-413. DOI: $10.1007 / 978-3-030-12082-5 \_37$

[23] Straumal B., Baretzky B., Mazilkin A., Protasova S., Myatiev A., Straumal P. // J. Eur. Ceram. Soc. 2009. Vol. 10. P. 1963-1970. DOI: 10.1016/j.jeurceramsoc.2009.01.005

[24] Федосеев В.Б. // Нелинейная динамика. 2017. Т. 13. № 2. C. 195-206. [Fedoseev V.B. // Russ. J. Nonlin. Dyn. 2017. Vol. 13. N 2. P. 195-206.] DOI: $10.20537 /$ nd1702004

[25] Долинный А.И. // Высокомол. соедин. Сер. В. 2005. Т. 47. № 8. С. $1557-1562$.

[26] Магомедов М.Н. // ТВТ. 1990. Т. 28. № 6. С. 1112-1170. [Magomedov M.N. // High Temp. 1990. Vol. 28. N 6. P. 843 847.]

[27] Магомедов М.Н. // ЖТФ. 2010. Т. 80. Вып. 9. С. 141-145. [Magomedov M.N. // Tech. Phys. 2010. Vol. 55. N 9. P. 1373 1377.] DOI: $10.1134 / \mathrm{S} 1063784210090227$

[28] Шишулин А.В., Федосеев В.Б., Шишулина А.В. // Бутлеров. сообщ. 2017. Т. 51. № 7. С. 31-37.

[29] Li Y.C., Wang C.P., Liu X.J. // CALPHAD: Computer Coupling Phase Diagrams Thermochem. 2009. Vol. 33. P. 415-419. DOI: 10.1016/j.calphad.2008.12.007

[30] Смирнова Н.Н., Колякина Е.В., Кулагина Т.Г., Гришин Д.Ф. // Изв. РАН. Сер. хим. 2013. № 10. С. 2251-2257. [Smirnova N.N., Kolyakina E.V., Kulagina T.G., Grishin D.F. /] Russ. Chem. Bull. 2013. Vol. 62. N 10. P. 2251-2257.] DOI: $10.1007 / \mathrm{s} 11172-013-0325-5$

[31] Li M., Jia P., Sun X., Geng H., Zuo M., Zhao D. // Appl. Phys. A. 2016. Vol. 122. P. 266. DOI: 10.1007/s00339-016-9819-y

[32] Zhao D., Bo L., Wang L., Li S. // Mater. Res. Express. 2018. Vol. 5. P. 046508. DOI: 10.1088/2053-1591/aaba4a

[33] Шишулин А.В., Федосеев В.Б., Шишулина А.В. // ЖТФ. 2019. Т. 89. Вып. 9. С. 1420-1426. [Shishulina A.V., Fedoseev V.B., Shishulina A.V. // Tech. phys. 2019. Vol. 64. N 9. P. 1343-1349]. DOI: 10.1134/S1063784219090172.

[34] Магомедов М.Н. // ЖТФ. 2016. Т. 86. Вып. 5. С. 84-91. [Magomedov M.N. // Tech. Phys. 2016. Vol. 61. N 5. P. 722 729.] DOI: $10.1134 / \mathrm{S} 1063784216050145$ 
[35] Магомедов М.Н. // Письма в ЖТФ. 2016. Т. 42. Вып. 14. C. 94-102. [Magomedov M.N. // Tech. Phys. Lett. 2016. Vol. 42. N 7. P. 761-764.] DOI: 10.1134/S1063785016070245

[36] Han C.D., Chun S.B., Hahn S.F., Harper S.Q., Savickas P.J., Meunier D.M., Li L., Yancin T. // Macromolecules. 2008. Vol. 31. N 2. P. 394-402. DOI: $10.1021 / \mathrm{ma} 971309 \mathrm{e}$

[37] Ougizawa T., Walsh D.J. // Polymer J. 1993. Vol. 25. N 12. P. 1315-1318. DOI: 10.1295 /polymj.25.1315

[38] Bondi A. // J. Phys. Chem. 1964. Vol. 68. N 3. P. 441-451. DOI: $10.1021 / \mathrm{j} 100785 \mathrm{a} 001$

[39] Тагер А.А. Физикохимия полимеров. М.: Химия, 1978. C. $337-368$.

[40] $W u$ S. // J. Macromol. Sci. C. 1974. Vol. 10. P. 1-73. DOI: $10.1080 / 15321797408080004$

[41] Jia L., Shi B. // J. Macromol. Sci. B. 2011. Vol. 50. P. 1042 1046. DOI: $10.1080 / 00222348.2010 .497439$

[42] Fowkes F.M. Chem. Phys. Interface. / Ed. by American Chem. Society. Washington: D.C., 1965. P. 1-12.

[43] Шишулин А.В., Федосеев В.Б. // Неорган. материалы. 2019. Т. 55. № 1. С. 16-20. [Shishulin A.V., Fedoseev V.B. // Inorg. Mater. 2019. Vol. 55. N 1. P. 14-18.] DOI: $10.1134 / \mathrm{S} 0020168518050114$

[44] Advances in Memristors, Memristive Devices and Systems / Ed. by S. Vaidyanathan, C. Volos. Springer, Cham, 2017. 511 p. DOI: $10.1007 / 978-3-319-51724-7$

[45] Герасимова С.А., Михайлов А.Н., Белов А.И., Королев Д.С., Горшков О.Н., Казанцев В.Б. // ЖТФ. 2017. Т. 87. Вып. 8. С. 1248-1254. [Gerasimova S.A., Mikhaylov A.N., Belov A.I., Korolev D.S., Gorshkov O.N., Kazantsev V.B. // Tech. Phys. 2017. Vol. 62. N 8. P. 1259 1265.] DOI: $10.1134 / \mathrm{S} 1063784217080102$

[46] Gerasimova S.A., Mikhaylov A.N., Korolev D.S., Belov A.I., Antonov I.N., Gorshkov O.N., Kazantsev V.B. // Opera Medica et Physiolog. 2016. Vol. 2. N 2(3). P. 64-80. DOI: $10.20388 / \mathrm{OMP} 2016.00 \mathrm{~S} 2.005$ 